\title{
Solving the Caesar Problem Without Categorical Sortals
}

\author{
Nikolaj Jang Pedersen
}

Received: 21 March 2005/Accepted: 11 February 2009/Published online: 10 March 2009

(C) The Author(s) 2009. This article is published with open access at Springerlink.com

\begin{abstract}
The neo-Fregean account of arithmetical knowledge is centered around the abstraction principle known as Hume's Principle: for any concepts $X$ and $Y$, the number of $X$ 's is the same as the number of $Y^{\prime}$ 's just in case there is a 1-1 correspondence between $X$ and $Y$. The Caesar Problem, originally raised by Frege in $\S 56$ of Die Grundlagen der Arithmetik, emerges in the context of the neo-Fregean programme, because, though Hume's Principle provides a criterion of identity for objects falling under the concept of Number-namely, 1-1 correspondence-the principle fails to deliver a criterion of application. That is, it fails to deliver a criterion that will tell us which objects fall under the concept Number, and so, leaves unanswered the question whether Caesar could be a number. Hale and Wright have recently offered a neo-Fregean solution to this problem. The solution appeals to the notion of a categorical sortal. This paper offers a reconstruction of their solution, which has the advantage over Hale and Wright's original proposal of making clear what the structure of the background ontology is. In addition, it is shown that the Caesar Problem can be solved in a framework more minimal than that of Hale and Wright, viz. one that dispenses with categorical sortals. The paper ends by discussing an objection to the proposed neo-Fregean solutions, based on the idea that Leibniz's Law gives a universal criterion of identity. This is an idea that Hale and Wright reject. However, it is shown that a solution very much in keeping with their own proposal is available, even if it is granted that Leibniz's Law provides a universal criterion of identity.
\end{abstract}

\footnotetext{
N. J. Pedersen $(\bowtie)$

Department of Philosophy, Dodd Hall, University of California Los Angeles, 405 Hilgard Avenue, Los Angeles, CA 90095-1451, USA

e-mail: nikolaj@ucla.edu
} 


\section{The Caesar Problem}

An abstraction principle, as it is understood in the context of the neo-Fregean programme, is a principle of the form

$$
(\forall \alpha)(\forall \beta)(\Sigma(\alpha)=\Sigma(\beta) \leftrightarrow \alpha \sim \beta)
$$

where $\Sigma$ is a term-forming operator taking as inputs expressions of the type $\alpha$ and $\beta$ and $\sim$ is an equivalence relation on entities denoted by expressions of that type. Neo-Fregeanism, championed by Bob Hale and Crispin Wright, is an attempt to provide an account of classical mathematics based on abstraction principles. ${ }^{1}$

In the case of arithmetic, neo-Fregeans appeal to what is known as Hume's Principle in the literature:

$$
(\forall X)(\forall Y)\left(\sharp X=\sharp Y \leftrightarrow X \approx_{1-1} Y\right)
$$

i.e. for any concepts $X$ and $Y$, the number of $X^{\prime}$ 's is the same as the number of $Y^{\prime}$ s if and only if there is a 1-1 correspondence between $X$ and $Y{ }^{2}$ The axioms of Peano arithmetic are interpretable in the system obtained by adding Hume's Principle to pure second-order logic. This result is known as Frege's Theorem.

According to the neo-Fregean, concepts introduced by abstraction principles should be genuinely sortal. That is to say, the concept should have a criterion of application and a criterion of identity. The criterion of application tells us what objects fall under the concept, while, among these, the criterion of identity distinguishes one from another. The Caesar Problem arises in the context of the neoFregean programme, because, though abstraction principles provide a criterion of identity for objects falling under the concept introduced, they do not in any direct way supply a criterion of application. ${ }^{3}$ In particular, Hume's Principle supplies a criterion of identity for numbers (of concepts) - namely, 1-1 correspondence-but fails to deliver a criterion of application. In other words, while the principle yields an answer to the question whether any two numbers are identical, it leaves unanswered which objects the numbers are. Consequently, it leaves unanswered the question whether Caesar could be a number. Of course, it strikes us as obvious that Caesar could not be a number. The problem for the neo-Fregean, however, is to tell a philosophical story that will entitle her to hold that this is so.

Before I proceed to a presentation of the Hale-Wright solution to the Caesar Problem, let me offer a few words on the intended scope of the paper. The project to be pursued is internal in nature. The basic ontology of Hale and Wright-a sortalbased ontology with certain features-will be assumed throughout. In making this assumption, I do not mean to suggest that sortal ontology is uncontroversial, or that there are no alternatives to it. I allow myself to make the assumption, however, because the aim of the paper is not to convince someone who does not favour a

\footnotetext{
${ }^{1}$ For more on the neo-Fregean programme see Hale and Wright (2001a), a collection of essays that develop the programme and respond to various criticisms launched against it.

${ }^{2} X \approx_{1-1} Y$ is definable in pure second-order logic: $X \approx_{1-1} Y=_{d f} .(\exists R)(\forall x)(X x \rightarrow(\exists y)(Y y \wedge$ $(\forall z)(R x z \rightarrow z=y)) \wedge(Y x \rightarrow(\exists y)(X y \wedge(\forall z)(R z x \rightarrow z=y))))$.

3 Hale and Wright (2001b, p. 368).
} 
sortal-based approach to ontology and a neo-Fregean solution the Caesar Problem that she should do so. Instead the aim is to shed light on Hale and Wright's solution to the Caesar Problem by presenting a version of their solution which makes it clearer what the structure of the underlying ontology is and to show how a substantial existence assumption can be eliminated without thereby losing the solution to the Caesar Problem. This project is worthwhile pursuing, because it can help facilitate a better understanding of a recent-and prominent-solution to the Caesar Problem. Furthermore, it sheds light on the structure of the sortal ontology, which, to my knowledge, is a matter that is not often explicitly discussed in the literature on sortal-based approaches to ontology.

\section{The Hale-Wright Solution to the Caesar Problem}

Hale and Wright concede that abstraction principles do not in any direct way supply a criterion of application for the concepts they serve to introduce. However, they maintain that, if developed properly, the framework of sortal concepts and criteria of identity does provide a solution to the Caesar Problem (Hale and Wright 2001b).

The basic strategy of Hale and Wright is to tell a story about the structure of neoFregean ontology, which will make it clear that there is just no chance that Caesar is - indeed, could be-a number. In brief outline, the ontology they envision is one in which

... all objects belong to one or another of a smallish range of very general categories, each of these subdividing into its own respective more or less general pure sorts; and in which all objects have an essential nature given by the most specific pure sort to which they belong. Within a category, all distinctions between objects are accountable by reference to the criterion of identity distinctive of it, while across categories, objects are distinguished by just that - the fact that they belong to different categories. It is surely because we already inchoately think in terms such as these that it strikes us as just obvious that Caesar is no number (Hale and Wright 2001b, pp. 390-391).

An object's falling under a given pure sort-or pure sortal concept-is a necessary feature of the object. If $x$ falls under a pure sortal $F$, it does so necessarily, and could not survive were it to cease to be $F$. ${ }^{4}$ The concepts of Number and Person are examples of pure sortals. Pure sortals contrast with a functional concept like Doorstop. If an object falls under a functional concept, its doing so need not be a necessary feature of it.

A reasonable reconstruction of the essence of the Hale-Wright solution to the Caesar Problem goes like this (where $F$ and $G$ are used for pure sortal concepts, as opposed to arbitrary concepts).

\footnotetext{
${ }^{4}$ Hale and Wright (2001b, p. 387).
} 
All objects of the ontology fall under sortal concepts, each of which is assumed to have a unique criterion of identity. ${ }^{5}$ Among the sortals we find categories, which are maximally extensive sortals in the sense that (i) all sub-sortals of a given category $F$ share their criterion of identity and (ii) for any object $x$, if $x$ is not $F$, then any sortal $G$ which $x$ falls under does not share its criterion of identity with $F{ }^{6}$ Internally, objects are unified in a category by falling under sortals that share their criterion of identity, while objects of distinct categories differ in exactly that respect, viz. that any sortals they fall under do not share their criterion of identity. Once this picture is in place, it can be shown that any two categories $F$ and $G$ are either co-extensional or have no object in common. (More on this later in this section and in the Appendix.) Since Caesar and numbers belong to different categories, Caesar cannot be a number.

Below I will spell this reasoning out in greater detail. I will offer rigorous definitions of the key notions and state the principles relied on in the reasoning. Five definitions and three principles will be stated. Two of the definitions serve to introduce an alternative to the characterization of a category adopted by Hale and Wright. The alternative characterization is provided for two reasons. Firstly, it makes it explicit that categories are equivalence classes under the relation '....shares criterion of identity with...' and so sheds light on the structure of the ontology underlying the Hale-Wright solution to the Caesar Problem. Secondly, the alternative characterization will be useful for the purposes of making the point that the neo-Fregean can do away with categories and still succeed in banning imperial inhabitants from the realm of abstracta.

These things being said, let me proceed to fill in the details of the reconstructed Hale-Wright solution. First, it should be said what it is for two sortals to share their criterion of identity. I will assume, with Hale and Wright, that every sortal has a unique criterion of identity. As usual 'iff' abbreviates 'if and only if'. ' $\square$ ' denotes metaphysical necessity, and ' $e q_{F}$ ' and ' $e q_{G}$ ' denote the equivalence relations giving the criterion of identity of respectively $F$ and $G$ :

Definition 1 (Sharing criterion of identity) Two sortals $F$ and $G$ share their criterion of identity iff $\square(\forall x)(\forall y)\left(x e q_{F} y \leftrightarrow x e q_{G} y\right)$. This will be written 'Share ${ }^{=}(F, G)$ '.

That is, two sortals $F$ and $G$ share their criterion of identity just in case, as a matter of metaphysical necessity, any two objects are related by the equivalence criterion giving the criterion of identity for $F$ just in case they are related by the

\footnotetext{
5 There is a substantial literature on the notion of a sortal and the related notion of a criterion of identity, including Wiggins' (2001) influential work. Certainly some authors differ greatly from Hale and Wright in their understanding of these notions and their range of applicability. See, e.g., Ayers (1974) and Geach (1962, 1967). However, it is not my concern here to discuss the merits of the various views on these matters. Throughout the paper I will assume the Hale-Wright view, as announced at the end of Sect. 1.

6 Dummett (1973, p. 76), Hale and Wright (2001b, p. 389). As stated by Hale and Wright, the second condition of the characterization is ambiguous between an existential and a universal statement. According to the former statement if an object does not fall under category $F$, then there is some sortal under which it falls which does not share its criterion of identity with $F$. Here I have chosen to characterize the notion of a category in terms of the latter, stronger statement since this is clearly what Hale and Wright need in order to put the troublesome emperor to rest.
} 
equivalence criterion giving the criterion of identity for $G$. Note that Share ${ }^{=}$is an equivalence relation on sortals. ${ }^{7}$

Let ' $F \subseteq G$ ' be shorthand for ' $(\forall x)(F x \rightarrow G x)$ ' and define sortal inclusion as follows:

Definition 2 (Sortal inclusion) For any two sortals $F$ and $G, F$ is sortally included in $G$ iff $F \subseteq G$ and $\operatorname{Share}^{=}(F, G)$. This will be written ' $F \preceq G$ '.

The definition says that $F$ is sortally included in $G$ (or is a sub-sortal of $G$ ) just in case $F$ and $G$ share their criterion of identity and every $F$ is $G$.

In giving Definition 1 , it was assumed that the equivalence relation $e q_{F}$ that yields the criterion of identity for a sortal $F$ can hold between objects that are not $F$. This will happen whenever $F$ is sortally included in $G$-i.e. $F \preceq G$-and there are identical $G$ 's that are not $F$. For in that case any such $G$ 's will be related by the equivalence relation that gives the criterion of identity for $F$, but will not be $F$ 'sand so, will not be the same $F$. As a consequence, $e q_{F}$ cannot be characterized as specifying what it takes for two objects whatsoever to be the same $F$, but must instead be characterized as specifying what it takes for two $F^{\prime}$ 's to be the same. ${ }^{8}$

As Definition 2 implies that $(\forall F)(\forall G)\left(F \preceq G \rightarrow\right.$ Share $\left.^{=}(F, G)\right)$, the DummettHale-Wright characterization of a category mentioned earlier can be simplified to:

Definition 3 (Category) A sortal $F$ is a category iff $(\forall x)(\neg F x \rightarrow(\forall G)(G x \rightarrow$ $\neg$ Share $\left.^{=}(F, G)\right)$. This will be written 'Cat $(F)$ '.

If a sortal $F$ is a category, it is maximally extensive in the sense that there can be no sortal $G$ which includes something $F$ does not include and shares its criterion of identity with $F$.

As noted, Share ${ }^{=}$is an equivalence relation on sortals. The equivalence class of a sortal $F$ under Share ${ }^{=}$is defined in the obvious way:

Definition 4 (Equivalence class under Share $^{=}$) The equivalence class of a sortal $F$ under Share $^{=}$is the class of sortals it is related to by Share $=$i.e. $|F|_{\text {Share }^{=}}=\left\{G:\right.$ Share $\left.^{=}(F, G)\right\}$.

Introduce the notion of a categorical sortal as follows:

Definition 5 (Categorical sortal) A sortal $F$ is a categorical sortal iff it is coextensional with the union of its equivalence class under Share $=$, i.e. $F$ is a categorical sortal iff for any $x, x$ falls under $F$ just in case $x$ falls under a sortal in $|F|_{\text {Share }}=$.

Definition 5 is the alternative characterization of the notion of a category presented above. In order to show that Definition 5 does indeed provide a faithful,

\footnotetext{
7 This can be verified by straightforward reasoning and without making any substantial assumptions about the nature of metaphysical necessity. Now, it will be a matter of metaphysical necessity that Caesar is not a number. This is something that can be known a priori. It can be known a priori, because it can be known through reflection on our concepts. Reflection on sortal concepts can serve as a guide to metaphysical matters, because these concepts indicate real divisions in the ontology. In my understanding of these matters I rely on conversations with Wright.

8 Thanks to a referee for making this point.
} 
alternative characterization of the notion of a category we need to show the following:

Theorem 1 For any sortal $F, F$ is a category if and only if $F$ is a categorical sortal.

The proof of Theorem 1 is straightforward and appears in the Appendix. In light of Theorem 1, I will allow myself to switch freely between talk of categories and categorical sortals.

What is gained by introducing the notion of a categorical sortal? An immediate advantage of using Definitions 4 and 5 instead of Definition 3 is that the structure of neo-Fregean ontology is made clearer. Definition 4 makes it explicit how sortals bundle up in equivalence classes under Share $^{=}$, and Definition 5 ties categorical sortals intimately to these equivalence classes. Another advantage of speaking explicitly about Share $=$ being an equivalence relation is that this, rather than the maximal extensiveness of categories, is what does all the work in the solution to the Caesar Problem. This point will be argued in Sect. 3.

We now move on to state the three principles needed for the Hale-Wright solution to the Caesar Problem. The first principle is the following:

Principle 1 Share ${ }^{\text {is }}$ an equivalence relation on sortals.

Strictly speaking, there is no need to state this as a separate principle, becauseas noted earlier-it follows by straightforward reasoning and without making any substantial assumptions about the nature of metaphysical necessity.

The second principle is one to the effect that if two sortals overlap-i.e. have an object in common - then they share their criterion of identity. That is,

Principle 2 For any sortals $F$ and $G$, if $(\exists x)(F x \wedge G x)$ then $\operatorname{Share}^{=}(F, G){ }^{9}$

Principle 2 is equivalent to the statement that no object can fall under sortals with distinct criteria of identity-let it be Principle $2^{\prime}$. Principles 2 and $2^{\prime}$ are similar to the principle (U) of Hale and Wright (see 2001b, p. 393) according to which no object can belong to more than one category. However, they are more general in the sense that (U) makes explicit reference to categories, while Principles 2 and $2^{\prime}$ can be incorporated into a framework without categories, as will be done in Sect. 3. I adopt Principle 2 here, because in their brief discussion of (U) Hale and Wright in effect implicitly endorse it. The principle will play an important role later.

The last principle reads as follows:

Principle 3 Let $X_{1}, \ldots, X_{\alpha}$ ( $\alpha$ some ordinal) be sortals such that any two of them share their criterion of identity. Then there is a sortal co-extensional with their union.

\footnotetext{
9 Hale and Wright have themselves suggested the following principle (in personal correspondence):

(*) For any sortals $F$ and $G$, if $(\exists x)(F x \wedge G x)$ then $(\exists H)(H \preceq F \wedge H \preceq G)$

- and, indeed, an earlier version of the paper made use of (*). However, as an anonymous referee observed, the slightly weaker Principle 2 is all that is needed by the neo-Fregean. Principle 2 follows from (*) and the fact that Share ${ }^{*}$ is an equivalence relation.
} 
Hence, whenever we have a range of sortals with the same criterion of identity, there is a sortal which they are all sortally included in. In particular, Principle 3 ensures that there is a sortal which is co-extensional with the union of each equivalence class under Share $=$. These sortals are, by Definition 5, categorical sortals. ${ }^{10}$

This completes the list of definitions and principles, and we shall now turn to the neo-Fregean solution to the Caesar Problem.

Let us start by recording a general fact about equivalence classes: any two equivalence classes under a given equivalence relation are either identical or disjoint. In particular, any two equivalence classes under Share ${ }^{=}$are either identical or disjoint-i.e. either such equivalence classes contain exactly the same sortals or have no sortal in common. This tells us something at the level of sortals, but, it is recalled, the Caesar Problem poses a question of identity at the level of objects. Fortunately, given the way we have defined categorical sortals, we obtain an analogous result at the level of objects:

Theorem 2 For any two categorical sortals $F, \quad G, \quad(\forall x)(F x \leftrightarrow G x)$ or $\neg(\exists x)(F x \wedge G x)$.

That is any categorical sortals $F$ and $G$ are either co-extensional or have no object in common. The proof of this result is straightforward and appears in the Appendix. (Given Theorem 1, an immediate corollary is that, for any categories $F$, $G,(\forall x)(F x \leftrightarrow G x)$ or $\neg(\exists x)(F x \wedge G x)$.)

Theorem 2 is of interest to us here, because it is a big step towards a neo-Fregean solution to the Caesar Problem. Its significance is captured in what I will refer to as 'The Conditional Result':

The Conditional Result If the (unique) criterion of identity associated with respectively the sortal concept of Number and that of Person are distinct, then no number can be a person.

Hale and Wright take it that the antecedent obtains. ${ }^{11}$ On this assumption it is straightforward to reason to the consequent.

Assume that the criteria of identity associated with respectively the sortal concept Number and the sortal concept Person are distinct. By Definition 4, Number and Person belong to different equivalence classes under Share $=$. By Definition 5 and Principle 3, there is a categorical sortal corresponding to each of these equivalence classes. Let these categorical sortals be $F$ and $G$. Number is sortally included in one

\footnotetext{
${ }^{10}$ Principle 3 is restricted to sortals that share their criterion of identity. If the result of taking arbitrary unions of sortals were a sortal, the neo-Fregean would be committed to a single super-sortal-i.e. the sortal co-extensive with the union of all sortals in the ontology. Hale and Wright deny that there is such a sortal (Hale unpublished note). For further discussion of an intimately related matter, see Sects. 4 and 5.

11 Hale and Wright (2001b, p. 389).
} 
of these and Person in the other. Since $F$ and $G$ are distinct categorical sortals, we can, by Theorem 2 , conclude that Caesar cannot be a number. ${ }^{12}$

\section{Doing Away with Categories}

In the previous section, a reconstruction of the Hale-Wright solution to the Caesar Problem was provided, offering along the way an alternative characterization of the notion of a category, which-it is hoped-serves to make the structure of the ontology envisioned by Hale and Wright fairly clear.

Concerns may remain. A potential source of concern is the reliance on categorical sortals. Someone might be sympathetic to the idea of a sortal-based ontology, but be reluctant to buy into the existence of categorical sortals. Is there any way to satisfy a person with this kind of reservation? It turns out there is. One can simplify the framework from the previous section by dropping the assumption that there are categorical sortals and still provide a solution to the Caesar Problem. Here goes:

Adopt the framework from the previous section, but leave out Definition 5 and Principle 3, i.e. suppose that we do away with categorical sortals. Why does this more minimal framework hold a solution to the Caesar Problem? The reason is this: maximal extensiveness is not essential to the proposed solution. What matters is that Share ${ }$ is an equivalence relation on sortals. Once this relation is in place, sortals bundle up in equivalence classes under the relation. Any two equivalence classes under Share ${ }^{=}$will either be identical or disjoint, i.e. contain the exact same sortals or have no sortal in common. Since criteria of identity are assumed to be unique, no sortals with distinct criteria of identity can be in the same equivalence class under Share $=$.

Having observed this, suppose that the antecedent of the Conditional Result is granted, as do Hale and Wright. We have already seen that, within their framework, this implies that Number and Person are sortally included in distinct categories. In our trimmed-down version of the framework, we are not entitled to hold this-since we have left categories behind. By Definition 4, we are, however, entitled to hold that the sortals Number and Person are in different equivalence classes under Share ${ }^{=}$. By Principle 2 (or $2^{\prime}$ ) it now follows that Caesar cannot fall under the concept of Number, as desired. ${ }^{13}$

\footnotetext{
${ }^{12}$ Note that the reasoning only goes through because Principle 2 has been assumed. The principle ensures that objects are rooted in exactly one category by obstructing the following line of thought: 'Your result that any two categories are either co-extensional or have no object in common is no good. It might just be that Caesar falls under sortals with distinct criteria of identity and can thus be in two categories after all.' Some people would certainly find Principle 2 objectionable. Whether or not Principle 2 is legitimate pertains to the question what is the proper characterization of the notion of a sortal and the related notion of a criterion of identity. Though this is an interesting question, it is not one I will engage with here, as announced in an earlier footnote.

${ }^{13}$ Someone might object that the solution just given only goes through because of the extra generality packed into Principle 2 compared to the principle (U) of Hale and Wright. However, as said earlier, Principle 2 merely makes explicit a commitment already implicit in Hale and Wright in their endorsement of $(\mathrm{U})$
} 


\section{All Under One?}

In this section, I will discuss a worry about the two approaches to the Caesar Problem presented in Sects. 2 and 3. The worry emerges when we consider the question whether there is a single criterion of identity which, for any two objects whatsoever, determines whether they are identical or not. The worry can be spelled out as a two-step argument. The first step makes it clear why it would be problematic if there were a single criterion by appeal to which all questions of identity could be settled. The second step of the argument aims to show that there is indeed such a criterion of identity.

Let us turn to the first step: why would it be a problem for the solutions to the Caesar Problem proposed above if there were a single criterion of identity which, for any objects whatsoever, determined whether they are identical or not? To answer this question let us take a step back and reflect on the basic idea behind the HaleWright and the 'no-categories' solutions-namely, that two objects are only eligible for being identical provided that they fall under sortal concepts that share their criterion of identity. According to this picture, if the sortal concepts of Person and Number do not share their criterion of identity - as the neo-Fregean holds-Caesar cannot be a number. However, if there were a single criterion of identity by appeal to which all questions of identity could be settled, this line of thought would be undermined. For in that case Caesar would be eligible for being identical to a number by the lights of the neo-Fregean. ${ }^{14}$

Let us now turn to the second step of the argument. The target conclusion is that there is a single criterion of identity, supplied by Leibniz's Law, by appeal to which all questions of identity can be settled. According to Leibniz's Law, any two objects are identical just in case they share all their properties. This principle is taken as a definition of identity (for first-order objects) in standard second-order logic. ${ }^{15}$ It can be formalized as follows:

$$
\text { (LL) } \quad(\forall x)(\forall y)(x=y \leftrightarrow(\forall X)(X x \leftrightarrow X y))
$$

where the range of the second-order quantifier is all properties on the first-order domain. (That is, the range of the second-order quantifier is the arbitrary subclasses of the first-order domain.)

The relation of sharing all properties is an equivalence relation on objects. Let this equivalence relation be denoted by ' $e q_{U}$ ' and consider the claim that it provides a universal criterion of identity, i.e. a criterion which, for any objects whatsoever,

\footnotetext{
${ }^{14}$ Hale expresses the view that the existence of a single, all-inclusive category would be bad news for the neo-Fregean (Hale unpublished note), but does not spell out why this is so. He also does not offer any reason why, on the Hale-Wright view, there should be no such a category. The former matter has just been addressed. The latter matter will not be addressed here. I will, though, discuss a related matter in Sect. 5 where it is argued that, after a fashion, a solution to the Caesar Problem is available even if there is a universal criterion of identity and, so, a single all-compassing category.

15 Shapiro (1991, p. 63). It is interesting to note that, having stated the definition, Shapiro continues to say, 'I do not intend to assert a deep philosophical thesis about identity with this definition.' As an anonymous referee remarks, this is presumably because $x=y$ and $(\forall X)(X x \leftrightarrow X y)$ are provably equivalent in standard second-order logic. On other the hand, the objection to be rehearsed below does take Leibniz's Law to be a deep philosophical thesis about identity.
} 
will allow us to determine whether or not they are identical. Now, since (LL) is a definition, it should hold of metaphysical necessity, i.e.

$$
(\mathrm{LL} \square) \quad \square(\forall x)(\forall y)(x=y \leftrightarrow(\forall X)(X x \leftrightarrow X y))
$$

or, using the abbreviation for the relation of sharing all properties, $\square(\forall x)(\forall y)\left(x=y \leftrightarrow x e q_{U} y\right)$.

Recall that, for a sortal $F$, there is a principle of the following form:

$$
(\forall x)(\forall y)\left(x=y \leftrightarrow x e q_{F} y\right)
$$

where the right-hand side of the embedded bi-conditional gives the criterion of identity for $F$. Sortals are, in part, individuated by their criterion of identity, and (1) can thus reasonably be strengthened to:

$$
(1 \square) \quad \square(\forall x)(\forall y)\left(x=y \leftrightarrow x e q_{F} y\right)
$$

Finally, recall that two sortals $F$ and $G$ share their criterion of identity just in case

$$
\square(\forall x)(\forall y)\left(x e q_{F} y \leftrightarrow x e q_{G} y\right)
$$

Thus, in order to show that $e q_{U}$ is a universal criterion of identity what needs to be established is that, for any criterion of identity $e q_{F}$ of a sortal $F$, $\square(\forall x)(\forall y)\left(x e q_{F} y \leftrightarrow x e q_{U} y\right)$.

Given the principles just stated, one can argue as follows: consider an arbitrary sortal $F$, arbitrary objects $x$ and $y$, and suppose that $x e q_{F} y$. By (1 $\square$ ), $x=y$-and so, by (LL $\square$ ), $x e q_{U} y$. Hence, $x e q_{F} y \rightarrow x e q_{U} y$. Conversely, suppose that $x e q_{U} y$. Then $x=y$, by (LL $\square$ )—whence $x e q_{F} y$, by (1 $\square$ ). Thus, $x e q_{U} y \rightarrow x e q_{F} y$. Combining these results, we get $x e q_{F} y \leftrightarrow x e q_{U} y$. Since $x$ and $y$ were arbitrary, we get $(\forall x)(\forall y)$ ( $x e q_{F} y \leftrightarrow x e q_{U} y$ ). The principles relied on in the reasoning hold as a matter of metaphysical necessity, and thus, we obtain $\square(\forall x)(\forall y)\left(x e q_{F} y \leftrightarrow x e q_{U} y\right)$.

In sum, contrary to what proponents of neo-Fregean solutions to the Caesar Problem maintain, there is a universal criterion of identity. The neo-Fregean solution-whether of the Hale-Wright or the no-categories kind-thus founders: whether we are dealing with people, numbers, or any other kinds of objects, Leibniz's Law provides the criterion of identity. ${ }^{16}$

\section{Concession Without Defeat: How to Recover the Solution to the Caesar Problem}

What is the status of Leibniz's Law within the neo-Fregean framework? Hale and Wright only address this question in passing. They say that whether or not Leibniz's Law 'offers a correct analysis of identity, it is clear that the idea of items' sharing all their (bona fide) properties cannot possibly serve as a working standard of identity. ${ }^{, 17}$ This suggests that they hold the view that, even if Leibniz's Law is true,

\footnotetext{
${ }^{16}$ Versions of the objection just rehearsed have been put to me by a number of people, including an anonymous referee.

17 Hale and Wright (2001a, b, p. 388).
} 
it is a further question whether it can provide a universal criterion of identity-and one which they think should be answered in the negative.

Hale and Wright present two arguments in support of a negative answer. However, they are both very condensed and rest on substantive assumptions that are left undefended. ${ }^{18}$ Fully unpacking the arguments would be quite the task. Instead of trying to execute it I will turn to a wholly different line of response-namely, a concessive one. The response starts by granting that Leibniz's Law provides a universal criterion of identity, but proceeds to argue that this does not undermine a solution to the Caesar Problem in keeping with the spirit of the solution offered originally. Dialectically this is a strong card for the neo-Fregean to hold. ${ }^{19}$

Now, to spell out the concessive line of response in detail, suppose that Leibniz's Law does indeed provide a universal criterion of identity and proceed to introduce a new, yet somewhat familiar-looking piece of terminology:

Definition $1^{*} \quad$ (Sub-sharing a criterion of identity) Two pure sortals $F$ and $G$ subshare a criterion of identity iff $\square(\forall x)(\forall y)\left(x e q_{F} y \leftrightarrow x e q_{G} y\right)$, where $e q_{F}$ and $e q_{G}$ are non-universal criteria of identity of respectively $F$ and $G$. This will be written 'Sub-Share' $(F, G)$ '.

In the present setting, the idea is that $\operatorname{Sub}_{\text {- }} \operatorname{sare}^{=}(F, G)$ holds just in case $F$ and $G$ share some criterion of identity less general than the one provided by Leibniz's Law.

Given Definition $1 *$, we obtain Principles $1 *-3 *$ and Definitions $4 *-5 *$ from Principles 1-3 and Definitions 4-5 by replacing occurrences of 'Share" with 'SubShare $^{=}$. Definition $4 *$ defines equivalence classes under Sub-Share ${ }^{=}$in the obvious way, while Definition $5^{*}$ introduces sub-categorical sortals as sortals that are coextensional with their equivalence class under Sub-Share ${ }^{=}$. Principle $1^{*}$ states that Sub-Share $^{=}$is an equivalence relation on sortals, while, according to Principle $2 *$, any sortals that have an object in common are related by Sub-Share ${ }^{=}$. Principle $2^{*}$ ensures that no object can fall under sortals that do not sub-share some criterion of identity. Principle $3 *$ says that there is a sortal co-extensional with the union of any sortals $X_{1} \ldots X_{\alpha}(\alpha$ an ordinal) related by Sub-Share $=$. This guarantees that there is a sub-categorical sortal for any equivalence class under Sub-Share ${ }^{=}$.

The following analogue of Theorem 2 can be established:

Theorem 2* For any two sub-categorical sortals $F, G,(\forall x)(F x \leftrightarrow G x)$ or $\neg(\exists x)(F x \wedge G x)$.

\footnotetext{
18 As can be verified by consulting Hale and Wright (2001b, p. 388).

19 In Sect. 1, it was stated that the Hale-Wright framework would be assumed throughout. One way of understanding this statement is that a sortal-based framework will be assumed throughout, and that the antecedent of the Conditional Result will be assumed throughout as well (i.e. that the (unique) criterion of identity associated with respectively the sortal concept Person and that of Number will be assumed to be distinct). Another, weaker way of understanding the statement is to take it to involve only the first assumption. What I intended was the weaker reading, and the argument given in Sect. 4 precisely challenges the antecedent of the Conditional Result by pushing the line that there is a universal criterion of identity. This seems to me a fair challenge, as Hale and Wright worry about there being a single allencompassing category - and relatedly, a universal criterion of identity-but never supply any argument against the existence of such a category.
} 
That is any sub-categorical sortals $F$ and $G$ are either co-extensional or have no object in common. This result can be established by modifying the proof of Theorem 2.

Theorem $2 *$ is relevant to our present concern-how to recover the neo-Fregean solution to the Caesar Problem. The significance of Theorem $2 *$ is captured in what we can call 'The Conditional Result*', a modification of The Conditional Result from Sect. 2:

The Conditional Result* If the sortal concept of Number and that of Person subshare no criterion of identity, then no number can be a person.

Provided that the antecedent obtains, the consequent easily follows:

Assume that Number and Person sub-share no criterion of identity. By Definition $4^{*}$, Number and Person belong to different equivalence classes under Sub-Share ${ }^{=}$. By Definition $5^{*}$ and Principle $3^{*}$, there is a sub-categorical sortal corresponding to each of these equivalence classes. Let these sub-categorical sortals be $F$ and $G$. Number is sortally included in one of these and Person in the other. Since $F$ and $G$ are distinct sub-categorical sortals, we can, by Theorem $2^{*}$, conclude that Caesar cannot be a number. ${ }^{20}$

\footnotetext{
${ }^{20}$ The following objection is due to an anonymous referee: Consider a model whose domain has as subsets the set of real numbers, the set of natural numbers and the set of people. The referee assumesreasonably, I grant- that, for each natural number and each person, there is a unique predicate that holds true of it. Now, interpret the second-order universal quantifier in Leibniz's Law as ranging over the subsets of the domain that serve as the semantic value of some unique predicate of the kind indicated, whether atomic or complex. Under this interpretation Leibniz's Law covers both natural numbers and people, and, yet, it is less general than Leibniz's Law without the indicated restrictions. Among other things, the unrestricted version provides a criterion of identity for real numbers, but the restricted version cannot (assuming that the language is countable). Hence, the attempted recovery fails: Number and Person sub-share a criterion of identity.

It seems to me that at least two lines of response are available. First, one can draw attention to the fact that, in the present dialectical setting, it has to be kept in mind that Leibniz's Law is also meant to supply a universal criterion of identity. For it was the worry about there being a universal criterion of identitysupplied by Leibniz's Law-that got us to the point where it might be thought attractive to pursue a recovery along the lines that I have suggested. To get Leibniz's Law on its intended interpretation-i.e. under the interpretation that will allow it to serve as a universal criterion of identity-the second-order quantifier needs to range over the full power set of the (first-order) domain. This interpretation of the second-order quantifier is the one given in standard semantics of second-order logic. Now, since the second-order quantifier is a logical item, it should receive the same interpretation in every model. If the standard interpretation is given across the board, and Leibniz's Law receives the intended interpretation, the worry just presented goes away. On the other hand, if the restricted interpretation of the second-order quantifier is given in every model, and Leibniz's Law is interpreted accordingly, then the worry about there being a universal criterion of identity goes away-and, with it, the need to attempt a recovery in the first place.
}

Second, one can draw attention to an idea which is integral to the sortal framework and which it is not clear that the objection can easily accommodate. The idea is this: criteria of identity must be associated with concepts that are pure sortals. An immediate question thus presents itself: what is the pure sortal concept that the restricted version of Leibniz's Law is associated with? For the unrestricted version of Leibniz's Law, the answer is 'Object' or 'Entity'. However, it is more difficult to provide an answer for the restricted version. Consider the suggestion that Number-or-Person is the relevant pure sortal. This seems implausible, because, upon reflection, the restricted version of Leibniz's Law should be able to serve as a criterion of identity for the class of objects that each has a property which is the semantic value of some (atomic or complex) predicate that applies uniquely to it. But what is the pure sortal that all these objects are meant to fall under? If there is a concept under which all the aforementioned objects fall, it is 
The Conditional Result* depends on Principle 2*. Principle $2 *$ ensures that objects are rooted in exactly one sub-categorical sortal. In the spirit of the nocategories solution presented earlier, it might be suggested that Principle $2^{*}$ enables us to recover the solution to the Caesar Problem in a framework that does not include Principle $3^{*}$. That is, in a framework that does away with sub-categorical sortals. In our present setting, this no-sub-categories proposal would commit one to accepting the existence of the universal, or all-inclusive, category, while rejecting the existence of sub-categories. There would be something slightly odd about that. Hence, if the concessive strategy is to be pursued, I recommend that it be pursued in its sub-categorical form.

Let me finish this section with some brief closing remarks on the recovered solution. The driving thought behind the solution is that, although there is a universal criterion of identity - and a corresponding equivalence class under Share $=$ containing every sortal-the internal structure of this equivalence class is still sufficiently divisive to keep numbers and people apart. The universal criterion of identity and the corresponding equivalence class may run against the letter of the original neo-Fregean proposal, as envisioned by Hale and Wright. However, it need not run against its spirit. For the structure of the ontology behind the recovered neoFregean solution very much preserves the features of the ontology behind the original solution. Sub-categorical sortals are the would-be categories of the Hale-

\section{Footnote 20 continued}

presumably a highly gerrymandered or artificial one - and it is a substantive issue whether such a concept can count as a pure sortal.

The referee also offers the equivalence relation $\simeq$ as a counterexample to the proposed solution, where $\simeq$ is defined as follows: $(\forall x)(\forall y)(x \simeq y \leftrightarrow$ any bijective mapping $f$ between the union of Person and Number and the von Neumann ordinal $\omega_{0}$ is such that $\left.f(x)=f(y)\right)$. $\simeq$ gives a criterion of identity for people as well as numbers-indeed, it would appear to do so for any pair of countable sortals. Yet, it does not deliver a criterion of identity for uncountable sortals and, so, is not a universal criterion of identity. Other logical or mathematical constructions can be carried out to deliver the same result.

When confronted with this supposed counterexample, adherents of sortal-based solutions to the Caesar Problem would harbour exactly the kind of reservation mentioned above. That is, they would not be convinced that the objection is a devastating one, because the objector faces several tasks. First, it must be specified what concept the equivalence relation that is meant to cause trouble serves as the criterion of identity for. Second, even if a concept can be specified, the further-crucial-issue remains whether it is a pure sortal. If, as would seem likely, the alleged trouble-making criteria of identity are paired with gerrymandered or artificial concepts, it is not clear that the neo-Fregean needs to worry.

Objectors may be dissatisfied with this kind of response and maintain that advocates of the proposed sortal-based solution cannot legitimately help themselves to assumptions about what kinds of concepts can enjoy a standing as pure sortals. These assumptions, they insist, must be argued for separately. I beg to differ. It seems to me that Hale and Wright-and others who favour a sortal-based approach to the Caesar Problem — can legitimately help themselves to the assumptions in question. At least they can do so in the context of this paper. For recall that the goal here is to execute a project that is internal to the neoFregean programme. The goal is not to convince the unconvinced. That is, this paper is not meant to convince those who are not already attracted to a broadly neo-Fregean approach to the Caesar Problem that such an approach is indeed the right one. Rather the task that I have set myself is to present a framework that makes the structure of neo-Fregean ontology clearer and also helps one to appreciate that a substantial existence assumption (the existence of categorical sortals) can be abandoned without undermining the prospects of a solution that is adequate from a broadly neo-Fregean point of view. The sortal-based framework is something that is taken as background, not something that is meant to be defended. Thus, although considerations on-or defences of-various aspects of sortal-based ontology are interesting and important, they are not part of the project that is being undertaken here. 
Wright framework in that they are the most general sortals, short of the universal, all-inclusive categorical sortal. They are so in the sense that, if $F$ is a sub-categorical sortal, there can be no other sortal $G$ such that $(\forall x)(F x \rightarrow G x)$, $\operatorname{Sub}_{\text {-share }}{ }^{(}(F, G)$, and $(\exists x)(\neg F x \wedge G x) .^{21}$

\section{Conclusion}

In Sects. 1-3, two things were accomplished. First, I presented a reconstruction of the Hale-Wright framework which makes it clear how their solution to the Caesar Problem works. In particular, Definition 5 served to introduce the notion of a categorical sortal, which sheds light on the structure of the ontology underlying the Hale-Wright solution by linking categorical sortals intimately to equivalence classes under Share ${ }^{=}$. Second, it was argued that a solution to the Caesar Problem can be given in a framework where no categorical sortals are assumed to exist. This is worth pointing out as some people may be sceptical about buying into the existence of categorical sortals though they are sympathetic to the idea of a sortalbased ontology. The lesson to be learned from this is the following: what matters to a sortal-based solution to the Caesar Problem is not maximal extensiveness, but rather the fact that sortals bundle up in equivalence classes under Share $=$.

Section 4 presented a challenge to the Hale-Wright and no-categories solutions to the Caesar Problem. The challenge was this: Leibniz's Law provides a universal criterion of identity, i.e. a criterion that will determine for any two objects whatsoever whether or not they are identical. The contention was that this undermines the proposed solutions, because they rely crucially on the assumption that the criteria of identity of Number and Person are distinct. In Sect. 5, I spelled out a concessive line of response to this challenge. It was granted that Leibniz's Law supplies a universal criterion of identity, and that there is a corresponding universal category. However, it was then argued that the internal structure of this universal category is very much in keeping with the ontology embraced by Hale and Wright. As a result, the concessive line of response supports a solution to the Caesar Problem which, although it does not conform to the letter of the initial neo-Fregean proposal, it does conform to its spirit.

Acknowledgements Thanks to Ben Caplan, Roy Cook, Philip Ebert, Bob Hale, Fraser MacBride, Carlotta Pavese, Agustín Rayo, Marcus Rossberg, Stewart Shapiro, Gabriel Uzquiano, Robbie Williams, Crispin Wright, and Elia Zardini for helpful discussion of earlier versions of this paper. I am particularly indebted to Bob Hale for discussion of Theorem 2. I am also indebted to two anonymous referees for a number of comments and criticisms that have improved the paper greatly.

Open Access This article is distributed under the terms of the Creative Commons Attribution Noncommercial License which permits any noncommercial use, distribution, and reproduction in any medium, provided the original author(s) and source are credited.

\footnotetext{
21 Since $\neg F x$ and $F$ is a sub-categorical sortal, $x$ does not fall under any sortal in $|F|$ Sub-share $=$. However, since $G x$ and Sub-share ${ }^{=}(F, G), x$ falls under some sortal in $|F|_{\text {Sub-share }^{=}}$. Contradiction.
} 


\section{Appendix: Proofs}

Theorem 1 For any sortal $F, F$ is a category if and only if $F$ is a categorical sortal.

Proof

$\rightarrow$ Suppose that $\operatorname{Cat}(F)$. To show: $F$ is a categorical sortal of its equivalence class

$|F|_{\text {Share }}$, i.e. for any $x, F x$ if and only if $x$ falls under a sortal in $|F|_{\text {Share }^{=}}$.

$\rightarrow$ Suppose that $F x$. By Definition 2, $\operatorname{Share}^{=}(F, F)$, whence, by Definition 4, $x$ falls under a sortal in $|F|_{\text {Share }}^{=}$, namely $F$.

$\leftarrow$ Suppose that $x$ falls under a sortal in $|F|_{\text {Share }}=-$ let it be $G$. For reductio assume $\neg F x$. Since $\operatorname{Cat}(F)$ and $G x, \neg$ Share $^{=}(F, G)$. Contradiction. So, $F x$.

$\leftarrow$ Suppose that $F$ is a categorical sortal. Suppose that $\neg F x$ and $G x$. Since $\neg F x$, by Definition 5, $x$ does not fall under a sortal in $|F|_{\text {Share }=}$. So, by $G x, G$ is not in

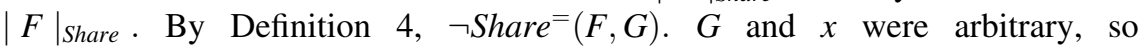
$(\forall x)\left(\neg F x \rightarrow(\forall G)\left(Y x \rightarrow \neg\right.\right.$ Share $\left.\left.^{=}\left(F, G^{=}\right)\right)\right)$.

Theorem 2 For any two categorical sortals $F, \quad G, \quad(\forall x)(F x \leftrightarrow G x)$ or $\neg(\exists x)(F x \wedge G x)$.

Proof For arbitrary categorical sortals $F$ and $G$ suppose, for reductio, that it is not the case that $(\forall x)(F x \leftrightarrow G x)$ or $\neg(\exists x)(F x \wedge G x)$, i.e. $\neg(\forall x)(F x \leftrightarrow G x)$ and $(\exists x)(F x \wedge G x)$. Since $(\exists x)(F x \wedge G x)$, Share ${ }^{=}(F, G)-$ by Principle 2. Now, $\neg(\forall x)$ $(F x \leftrightarrow G x)$, i.e. $(\exists x)((F x \wedge \neg G x) \vee(\neg F x \wedge G x))$. Suppose that $F x \wedge \neg G x$. Since $\neg G x$ and $G$ is a categorical sortal, $x$ does not fall under any sortal in $|G|_{\text {Share }=}$. So, since $F x, F$ is not in $|G|_{\text {Share }}=$, hence $\neg$ Share $\left(F^{=}, G^{=}\right)$. The other case is similar. Either way: $\neg$ Share $^{=}(F, G)$. Contradiction.

\section{References}

Ayers, M. (1974) Individuals without sortals. Canadian Journal of Philosophy, 4, 113-148.

Dummett, M. (1973). Frege's philosophy of language. London: Duckworth

Frege, G. (1884). Die Grundlagen der Arithmetik.

Geach, P. (1962). Reference and generality. Ithaca, NY: Cornell University Press.

Geach, P. (1967). Identity. Review of Metaphysics , 21, 3-12.

Hale, B. On the disjointness of categories, unpublished note.

Hale, B., \& Wright, C. (2001a). The reason's proper study. Oxford: Oxford University Press.

Hale, B., \& Wright, C. (2001b). To bury Caesar. In B. Hale \& C. Wright (Eds.), The reason's proper study (pp. 335-396). Oxford: Oxford University Press.

Shapiro, S. (1991). Foundations without foundationalism. Oxford: Oxford University Press.

Wiggins, D. (2001). Sameness and substance renewed. Cambridge: Cambridge University Press. 